\title{
Breves reflexiones sobre Lugar de Enunciación
}

\author{
Djamila Ribeiro*
}

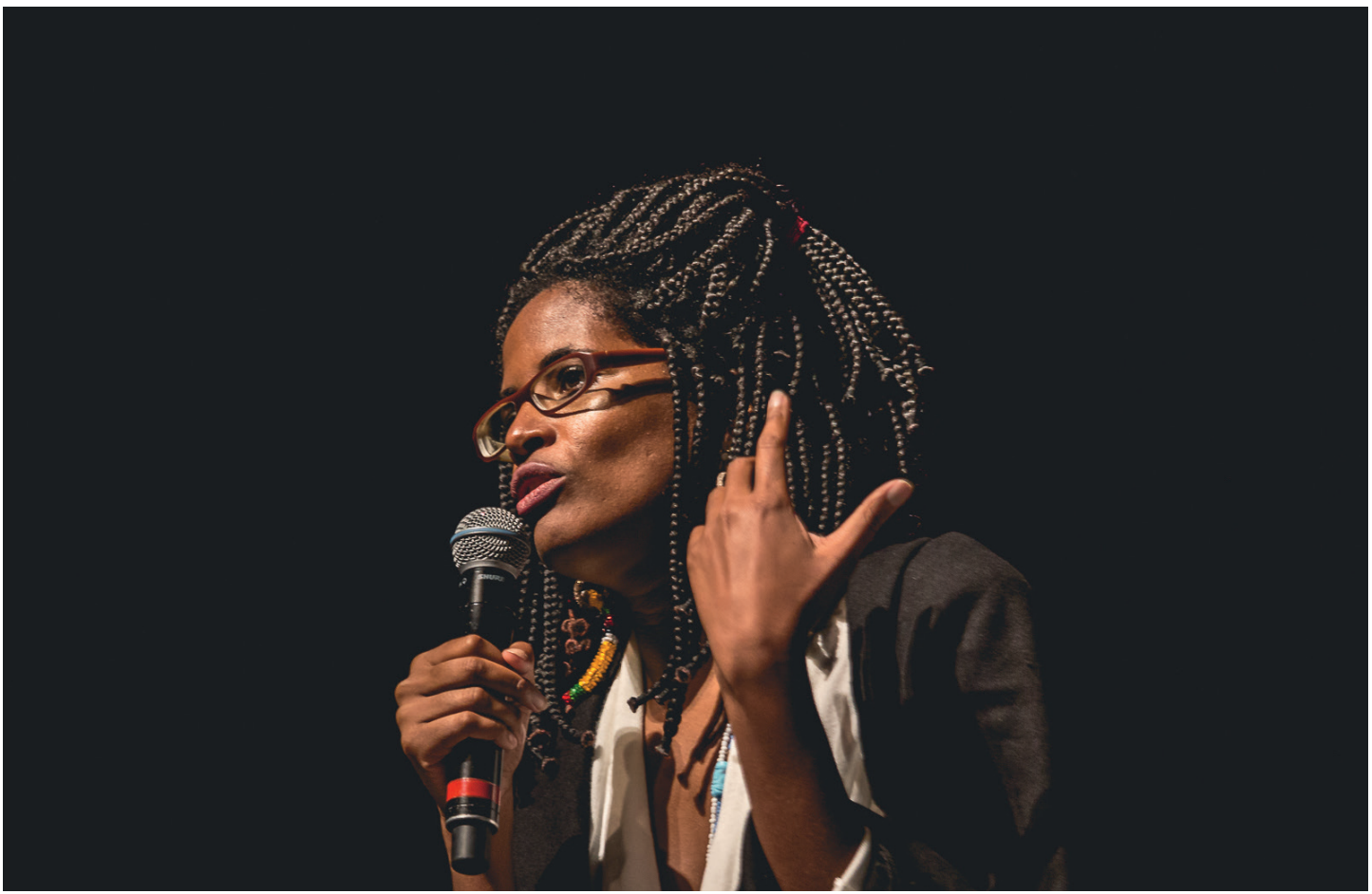

Foto: Luciano Viana/SESC

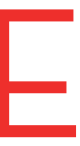

n noviembre de 2017, publiqué en Brasil el libro "¿O que é Lugar de Fala?" (¿Qué es Lugar de Enunciación?) ${ }^{1}$, primer título de la colección Feminismos Plurales, el cual reúne discusiones sobre conceptos de autores negros, sobre todo mujeres, con un precio muy accesible y lenguaje didáctico. Hasta el momento, fueron cinco títulos y más de cincuenta mil ejemplares vendidos en ferias de libros, librerías y universidades. En el ámbito universitario, incluso, se pasó a incluir ¿Qué es Lugar de Enunciación? entre las bibliografías de los cursos de Filosofía, Ciencias Sociales, Comunicación, etc., además de las otras obras de la colección. Es importante señalar, asimismo, que estos cambios son "anormales" en Brasil, dado que ese país fue el último de las Américas a abolir la esclavitud y que ostenta vergonzosos índices de desigualdades y violencias raciales estructurales.

Junto a otros autores y autoras - como Abdias do Nascimento, autor de la obra $O$ genocídio do negro brasileiro, y Sueli Carneiro, que en su tesis doctoral trabajó filosóficamente sobre el concepto epistemicidio- entendemos la categoría de violencia más allá de la violencia física realizada por el estado hacia los cuerpos,
*Djamila RIBEIRO, Filósofa, feminista y académica brasileña. Es investigadora y tiene un Máster en Filosofía Política por la Universidad Federal de São Paulo (Unifesp). En su trayectoria académica ha desarrollado investigaciones sobre las relaciones entre raza, género y feminismo. Organizadora de la colección Feminismos Plurales, también es columnista de la revista Carta Capital y exsecretaria Adjunta de Derechos Humanos y Ciudadanía de la ciudad de São Paulo durante la gestión de Fernando Haddad.

Traducción: Gabriela DE LIMA GRECCO

DOI: https://doi, org/10.15366/relacionesinternacionales2018.39.001

1 RIBEIRO, Djamila, O que é Lugar de Fala? Letramento, Belo Horizonte, 2017. 
realidad presente en Brasil contra grupos sociales vulnerables. Nos referimos, también, a otras formas de violencia, como la violencia cultural, económica y epistemológica, es decir, la invisibilización de saberes producidos lejos de la lógica eurocéntrica. La brillante intelectual brasileña Lélia González -feminista negra, profesora de la Pontificia Universidad Católica de Río de Janeiro, que falleció a mediados de la década noventa-, al percibir la jerarquización de conocimientos como producto de la clasificación racial de la población, destacó que quien posee privilegios sociales, posee el privilegio epistémico. Según la autora, el racismo se constituye "como la ciencia de la superioridad eurocristiana (blanca y patriarcal)". Esa reflexión de Lélia González nos proporciona pistas para entender el régimen de autorización discursiva, es decir, qué voces son legitimadas y qué voces no lo son².

En ese sentido, como se desprende de la tradición de estudios llevados a cabo por intelectuales negros y negras, al referirnos a grupos sociales oprimidos, es decir, a los que huyen de la norma hegemónica, es muy frecuente la deslegitimación e invisibilización de sus identidades y discursos. Sin embargo, la historia de resistencia y de lucha de las mujeres negras es larga, tanto en la producción de epistemologías como de sus propias existencias. En 1851, en la Convención de Derechos de la Mujer de Ohio, en Estados Unidos, Sojourner Truth -mujer esclavizada, nacida en cautiverio en Nueva York, y que se convirtió en abolicionista, escritora y activista de los derechos de la mujer- improvisó el discurso intitulado ¿Acaso no soy una mujer?. Este discurso fue registrado por Frances Gages, feminista y una de las autoras del gran compendio de materiales sobre la primera ola. Además de ser importante como evidencia de la insurgencia y disputa de narrativas de mujeres negras contra el modelo dominante, en el discurso de Sojourner, reproducido a continuación, es posible verificar el gran dilema que el feminismo hegemónico vendría a enfrentar: la universalización de la categoría mujer.

Bueno, niños. Donde hay tanto jaleo tiene que haber algo fuera de balance. Creo que con esa unión de negros del Sur y de mujeres del Norte, todos ellos hablando de derechos, los hombres blancos estarán en un aprieto bastante grande.

Pero ¿de qué están hablando todos aquí?

Ese hombre de allí dice que las mujeres necesitan ayuda al subirse a los carruajes, al cruzar las zanjas y que deben tener el mejor sitio en todas partes iPero a mí nadie me ayuda con los carruajes, ni a pasar sobre los charcos, ni me dejan un sitio mejor! ¿Y acaso no soy yo una mujer? iMiradme! iMirad mi brazo! He arado y plantado y cosechado, y ningún hombre podía superarme. ¿Y acaso no soy yo una mujer? Conseguí trabajar y comer igual que un hombre - cuando tuve para comer- y también aguanté los latigazos ¿Y no soy una mujer? He tenido trece hijos, y los vi ser vendidos a casi todos como esclavos, y cuando lloraba con el dolor de una madre inadie, sino Jesús, me escuchaba! ¿Y acaso no soy yo una mujer?

Ustedes hablan de esa cosa en la cabeza ¿Cómo es que le dicen? iEso es, cielo! Intelecto ¿Qué tiene que ver eso con los derechos de las mujeres o de los negros? Si mi copa no tiene espacio más que para una pinta, y la tuya para un cuarto de galón, ¿no es feo por tu parte no dejarme tener mi

2 RIBEIRO, Djamila, O que é..., op.cit., p. 25. 
pequeña media medida llena?

Entonces ese hombre pequeño de negro allá, él dice que las mujeres no podemos tener tantos derechos como los hombres, iporque Cristo no era una mujer! ¿De dónde viene tu Cristo? ¿De dónde viene tu Cristo? iDe Dios y de una mujer! El hombre no ha tenido nada que ver con Él.

Si la primera mujer que Dios hizo fue lo suficientemente fuerte para dar vuelta al mundo sola, estas mujeres juntas deben ser capaces de darle la vuelta al mundo en sí mismo iy ponerlo del lado correcto para arriba de nuevo! Y ahora que ellas piden hacerlo, ilos hombres mejor las dejan!

Agradecida de que me hayan escuchado, y ahora la vieja Sojourner no tiene nada más que decir.

Es decir, cuando hablamos de mujer, ¿de qué mujer estamos hablando? Como afirma la pensadora portuguesa, con ascendencia de Cabo Verde, Grada Kilomba, hay que complejizar la categoría de "otros" conceptualizado por Simone de Beauvoir. La mujer negra sería el "otro del otro", ya que ella es la doble antítesis de la blanquitud y la masculinidad. La investigadora señala, así, que la mujer blanca es mujer, pero es blanca; el hombre negro es negro, pero es hombre. Por otra parte, la mujer negra posee contra sí diferentes estructuras de opresión e invisibilidad histórica. En el libro ¿Qué es Lugar de Enunciación?, traigo reflexiones inspiradoras de Lélia González, tanto al comienzo como al final de la obra, que registran adecuadamente esa herencia histórica de lucha (y coraje) por disputar narrativas y existencias por mujeres negras:

Y el riesgo que asumimos aquí es el del acto de hablar con todas las implicaciones. Exactamente porque hemos sido hablados e infantilizados (infans es aquel que aún no puede hablar, el niño sin voz propia, a quien nos referimos en tercera persona, porque habla por medio de la interpretación de los adultos) es que en este trabajo asumimos nuestro propio discurso. Es decir, la basura va a hablar, y de buenas ${ }^{3}$.

La basura va a hablar, y de buenas. Al reivindicar el protagonismo y la presencia de la producción de sus saberes, el feminismo negro fundó las reflexiones derivadas de las intersecciones entre opresiones de raza, clase y género. La intelectual estadounidense Patricia Hill Collins es una fuente importante para profundizar el análisis aquí propuesto. De acuerdo con el dossier Mulheres negras: retrato das condições de vida das mulheres negras no Brasil, publicado por el Instituto de Investigación Aplicada en 2013,

El foco del feminismo negro es subrayar la diversidad de experiencias tanto de mujeres como de hombres, y los diferentes puntos de vista posibles de análisis de un fenómeno, así como marcar el lugar de enunciación de quien la propone. Patricia Hill Collins es una de las principales autoras de lo que se denomina feminismo del punto de vista. En su análisis, Collins (1990) lanza el concepto de matriz de dominación, para pensar la intersección de las desigualdades en la cual una misma persona puede encontrarse en diferentes posiciones, dependiendo de sus características. Así, el elemento

3 GONZALEZ, Lélia, "Racismo e sexismo na cultura brasileira" en Revista Ciências Sociais Hoje, 1984, pp. 223244. 
representativo de las experiencias de las diferentes formas de ser mujer estaría asentado en el entrecruzamiento entre género, raza, clase y generación, sin predominio de ningún elemento sobre otro (SOTERO, 2013, p. 36).

Collins identifica las experiencias compartidas que experimentan los negros por pertenecer a un grupo social específico. En Brasil, por ejemplo, según datos del Mapa de la Violencia, cada 23 minutos un joven negro es asesinado, muchos de ellos como consecuencia de la violencia policial. Asimismo, existen diversos estudios en Brasil que apuntan el menor uso de anestesia en partos y, a la vez, mayores índices de violencia obstétrica, en relación con las mujeres negras, representando, pues, una experiencia común para el grupo social. Es decir, el sólo hecho del individuo pertenecer a ese grupo social, independiente de su voluntad, ya lo hace susceptible de experimentar la truculencia por parte de las instituciones policiales. Se trata, por lo tanto, de un debate estructural.

No se trata, pues, de una forma de esencialización de la vivencia individual. En el artículo Comentário sobre o artigo de Hekman 'Truth and Method: Feminist Standpoint Theory Revisited: Onde está o poder? ${ }^{4}$, la investigadora Susan Hekman examina la teoría del punto de vista feminista y dice:

En primer lugar, la teoría del punto de vista se refiere a experiencias históricamente compartidas y basadas en grupos. Los grupos tienen un grado de continuidad a lo largo del tiempo de tal manera que las realidades de grupo trascienden las experiencias individuales. Por ejemplo, los afroamericanos, como un grupo racial estigmatizado, ha existido mucho antes de que yo naciera $y$, probablemente, continuará después de mi muerte. Aunque mi experiencia individual con el racismo institucional es única, las oportunidades y las limitaciones que me atraviesan diariamente serán similares a las que los afroamericanos se enfrentan como grupo. Argumentar que los negros, como grupo, van a transformarse o desaparecer basándome en mi participación, suena narcisista, egocéntrico y arquetípicamente posmoderno. En contraste, la teoría del punto de vista feminista enfatiza las condiciones sociales que constituyen estos grupos por sobre las experiencias individuales dentro de los grupos socialmente construidos.

La hipótesis defendida en la obra argumenta que, a partir de la teoría del punto de vista feminista, es posible hablar del lugar de enunciación. Como afirmo en mi obra:

En Brasil, comúnmente oímos ese tipo de crítica en relación con el concepto, porque los críticos parten de individuos y no de las múltiples condiciones que resultan en las desigualdades y jerarquías que ubican grupos subalternizados. Las experiencias de estos grupos ubicados socialmente de forma jerarquizada y no humanizada, hacen que las producciones intelectuales, los saberes y las voces sean tratadas de modo igualmente subalternizados, además de que las condiciones sociales los mantengan en un lugar silenciado estructuralmente.

4 COLLINS, Patricia Hill, "Comentário sobre o artigo de Hekman 'Truth and Method: Feminist Standpoint Theory Revisited': Onde está o poder?" en Signs, v. 22, n. 2, 1997, pp. 375-381 [Traducción de Juliana Borges]. 
Esto, en modo alguno, significa que esos grupos no creen herramientas para enfrentar esos silencios institucionales; por el contrario, existen varias formas de organización política, cultural e intelectual. La cuestión es que esas condiciones sociales dificultan la visibilidad y la legitimidad de esas producciones. Una simple pregunta que nos ayuda a reflexionar es: ¿a cuántas autoras y autores negros leyeron o tuvieron acceso durante el período de la graduación quienes cursaron la facultad? ¿Cuántas profesoras o profesores negros tuvieron? ¿Cuántos periodistas negros, de ambos sexos, existen en los principales periódicos del país o, incluso, en los medios alternativos?

Estas experiencias comunes, resultantes del lugar social que ocupan, impiden que la población negra acceda a ciertos espacios. En este sentido, podemos entender que es posible hablar del lugar de enunciación a partir del punto de vista feminista: no poder acceder a ciertos espacios supone no tener producciones y epistemologías de esos grupos en esos espacios; no poder estar de forma justa en las universidades, medios de comunicación, política institucional, por ejemplo, imposibilita que las voces de los individuos de esos grupos -incluso para quienes tienen acceso a internet- sean catalogadas o escuchadas. Hablar no se restringe al acto de emitir palabras, sino de poder existir. Pensamos lugar de enunciación como una forma de refutar la historiografía tradicional y la jerarquización de saberes consecuente de la jerarquía social ${ }^{5}$.

Dicho eso, hay que destacar que cuando hablamos del Lugar de Enunciación estamos hablando de locus social, es decir, del lugar social desde donde los grupos se originan. Por ello, de acuerdo con nuestra perspectiva, son equivocados los argumentos que tratan de silenciar debates o que subrayan que personas blancas no podrían teorizar sobre racismo, o que los hombres no podrían hacerlo tampoco en relación con el machismo. Es necesario que, cada vez más, hombres blancos-cis, por ejemplo, estudien blaquitud, cisgeneridad, masculinidad; que entiendan, a partir de una visión crítica, desde qué lugar social vienen, para pensar y existir en el mundo, incluso, garantizando una multiplicidad de voces y perspectivas de otros grupos invisibilizados. Además, se trata de una postura ética, puesto que, en nuestro entendimiento, no puede haber una desresponsabilización del sujeto con poder. A lo largo de la historia, ha sido subrayado por diversas intelectuales negras y negros la necesidad de romper con el régimen de autorización discursiva, con el silenciamiento y/o supresión de saberes, para combatir la violencia estructuralmente impuesta contra grupos oprimidos.

\section{Bibiografía}

RIBEIRO, Djamila, O que é Lugar de Fala? Letramento, Belo Horizonte, 2017.

CARNEIRO, Aparecida Sueli, A construção do outro como não-ser como fundamento do ser, Faculdade de Educação da Universidade de São Paulo, 2005 [Tesis doctoral].

GONZALEZ, Lélia, "Racismo e sexismo na cultura brasileira" en Revista Ciências Sociais Hoje, 1984, pp. 223-244.

COLLINS, Patricia Hill, "Comentário sobre o artigo de Hekman 'Truth and Method: Feminist Standpoint

5 RIBEIRO, Djamila. O que é Lugar de Fala?. Editora Letramento. p. 63/64 
Theory Revisited': Onde está o poder?" en Signs, v. 22, n. 2, 1997, pp. 375-381 [Traducción de Juliana Borges].

COLLINS, Patricia Hill, Black feminist thought: knowledge, consciousness and the politics of empowerment, Routledge, Nueva York - Londres, 1990.

SOTERO, Edilza Correia, "Transformações no acesso ao ensino superior Brasileiro: algumas implicações para os diferentes grupos de cor e sexo" en MAZZINI MARCONDES, Mariana; PINHEIRO, Luana; QUEIROZ, Cristina; QUERINO, Ana Carolina y VALVERDE, Danielle (Org.), Dossiê Mulheres negras: retrato das condições de vida das mulheres negras no Brasil, IPEA, Brasilia, 2013, pp. 35-52.

TRUTH, Sojourner, "Não Sou Uma Mulher?", 2009: https://goo.gl/1eQobC [Consultado el 10 de septiembre de 2018]. 


\section{RELACIONES INTERNACIONALES}

Revista académica cuatrimestral de publicación electrónica Grupo de Estudios de Relaciones Internacionales (GERI) Universidad Autónoma de Madrid, España

www.relacionesinternacionales.info

ISSN 1699 - 3950

ff facebook.com/RelacionesInternacionales

twitter.com/RRInternacional 